REGULAR PAPER

Second-harmonic generation from supported carbon nanotube films grown by chemical vapor deposition on fused silica

To cite this article: Satoru Okawara et al 2019 Jpn. J. Appl. Phys. 58032006

View the article online for updates and enhancements. 


\title{
Second-harmonic generation from supported carbon nanotube films grown by chemical vapor deposition on fused silica
}

\author{
Satoru Okawara ${ }^{1}$, Masafumi Kudo ${ }^{1}$, Mariko Miyamori ${ }^{1}$, Shinya Ohno ${ }^{1}$, Yoshihiro Shimazu ${ }^{1}$, Masatoshi Tanaka ${ }^{1} \oplus$, and \\ Takanori Suzuki ${ }^{1,2 *}$ (i) \\ ${ }^{1}$ Department of Physics, Faculty of Engineering, Yokohama National University, 79-5 Tokiwadai, Hodogaya, Yokohama 240-8501, Japan \\ ${ }^{2}$ Department of Applied Physics, School of Applied Sciences, National Defense Academy, 1-10-20 Hashirimizu, Yokosuka, Kanagawa 239-8686, Japan \\ *E-mail: tsuzuki@nda.ac.jp
}

Received October 11, 2018; revised December 31, 2018; accepted January 5, 2019; published online February 13,2019

Surface second-harmonic generation (SHG) is shown to be observable for carbon nanotube (CNT) films grown by CVD on fused silica. The SHG intensity ratio for incident radiation polarized perpendicular and parallel to the plane of incidence at the fundamental radiation (1064 $\mathrm{nm})$ is observed to have typical values for the two kinds of grown film: a film with aligned CNTs and a film with randomly oriented CNTs lying on a substrate sheet. SHG is discussed to originate not from the CNT bulk but from the film surface. The polarization ratio obtained for the two kinds of film was consistent with that calculated for a composite film of spheroidal particles with varying diameters employed to approximate the actual CNT films. The present findings may be applied to in situ evaluation of the initial stage in the CVD growth process of CNTs.

(C) 2019 The Japan Society of Applied Physics

\section{Introduction}

The nonlinear optics of carbon nanotubes (CNTs) have attracted considerable attention because of their quasi-onedimensional structures and unique electronic properties applicable to field effect transistors, diodes, gas sensors, transparent conductive materials and so on, which also suggests the possibility of their application in optical harmonic generation and ultrafast optics and photonics. ${ }^{1-5)}$ Single-walled carbon nanotubes (SWNTs) can be regarded as a rolled-up graphene sheet shaped as rather long cylindrical tubes with a diameter up to $2 \mathrm{~nm}$, and characterized by the tube diameter and chiral angle. The resulting low dimensionality (quasi-one-dimensional structure) determines most of the electronic properties relevant for the nonlinear optical response of SWNTs. From the structural point of view, SWNTs are classified into two types in accordance with their symmetry properties determined by the chiral indices $(n, m)$. Indices with combinations $(n, n)$, called armchair, and $(n, 0)$, called zigzag, determine invariant achiral SWNTs, whereas all other values of the indices $(n, m)$ give rise to chiral SWNTs which lack invariance under the inversion operation. Because the electronic structure of CNTs is either metallic or semiconducting depending on their chiral indices (metallic for $2 n+m=3 N$ and semiconducting for all other combinations), naturally formed CNTs may be a mixture of $1 / 3$ metal and 2/3 semiconducting CNTs. Here, $N$ and $n$ are positive integers, and $m$ is zero or a positive integer.

Currently, relatively few research works have addressed the second-harmonic generation (SHG) abilities of CNTs because the second-order nonlinear susceptibility $\chi(2)$ vanishes for centrosymmetric materials and racemic mixtures. This is in contrast to third-harmonic generation where there are no such restrictions. In CNT materials, it has been discussed that the optical second harmonics (SH) are generated through surface interactions, local imperfections, deformation or the macroscopic chirality resulting from the nonracemic assembly of the CNT structure. ${ }^{6-9)}$ From the observation that there was no SHG from commercially available CNTs, in contrast to the SHG observed for a sample grown with a catalyst-free method, together with other polarization measurements, the authors proposed that chiral SWNTs with strong structural defects are responsible for SHG. ${ }^{10,11)}$ All the SHG experiments to have studied SWNTs so far have attributed the origins of SHG to chirality or surface anisotropies or local deformations in the structure. $^{8-11)}$

Reference 6 reported SHG from well-aligned $0.4 \mathrm{~nm}$ single-walled chiral CNTs in the channels of $\mathrm{AlPO}_{4}-5$ zeolite. Since the dependence of $\mathrm{SH}$ polarization on excitation polarizations and the spectral feature with a resonance at $2 \mathrm{eV}$ were consistent with theoretical calculations of $\chi(2)$ for chiral $(4,2)$ CNT species, ${ }^{12)}$ the researchers were able to identify that the SHG originated from chirality. SHG from individual SWNTs has also been demonstrated by imaging individual tubes, and it was suggested that the structural noncentrosymmetry required for SHG arose from the nonzero chiral angle of the SWNTs. ${ }^{13)}$

CVD is currently the most popular method of producing CNTs. In CVD, thermal decomposition of a hydrocarbon vapor is achieved in the presence of a metal catalyst. ${ }^{2-5)} \mathrm{CVD}$ is a simple and economical technique for synthesizing CNTs at low temperature and ambient pressure. CVD is known to provide superior structural control of CNT architecture, and the obtained yield and purity are better than for the arc and laser methods. CVD is the only method that provides structural control. ${ }^{14,15)}$ The water-assisted synthesis method (super-growth CNT synthesis) has been demonstrated to produce super dense and vertically aligned nanotube forests with heights up to $2.5 \mathrm{~mm}$. ${ }^{16)}$

Although various techniques for producing CNTs have been proposed to improve the yield and the diameter distribution, the samples produced are still mixtures. ${ }^{17)}$ It is clearly very important to characterize the interfacial structure between SWNTs and the ambient air in order to control CNT growth for particular practical applications. Nonlinear optical spectroscopy is a powerful techniques for this structural characterization due to its high sensitivity to the interface. ${ }^{18)}$ Then, surface SHG or interface SHG may demonstrate the SHG capabilities that reflect the topologies and orientations of the CNTs. ${ }^{19)}$ Metallic or semiconducting CNT films, or chiral or racemic films, may also be expected to demonstrate 
different SHG capabilities. $^{20,21)}$ Moreover, an enhancing electric field (lightening rod) may also be expected in the needle-like structure of CNTs. ${ }^{19)}$

In the present study, we examined the surface SHG capability of CNT films on a supported sample prepared inhouse by CVD on a $\mathrm{SiO}_{2}$ substrate. The SHG intensity for incident radiation polarized perpendicular and parallel to the plane of incidence for the fundamental radiation $(1064 \mathrm{~nm})$ and the intensity ratio were observed to have typical values for the two kinds of grown film-aligned carbon nanotube (A-CNT) film and a film of randomly oriented CNTs lying on a substrate sheet (R-CNT) film-as evaluated by Raman and scanning electron microscopy. These values are also different from that of a smooth metal surface. By approximating the CNT-air interface of CNT films by a composite film of spheroidal particles with varying diameters, we were able to calculate and qualitatively reproduce the observed polarization ratios. ${ }^{22,23)}$ The SHG is thus discussed to originate not from the CNT bulk but from the film surface; moreover, we discuss that the surface morphology of the CNT film is reflected in the polarization ratio. The present finding may certainly be applied to in situ evaluation of the initial stage of CVD growth of CNTs.

\section{Experimental details}

\subsection{Sample preparation}

CNTs were grown on catalyst-coated substrates by ethanolCVD. The performance of metal catalysts is known to depend largely on the support material ${ }^{24)}$ and an alumina $\left(\mathrm{Al}_{2} \mathrm{O}_{3}\right)$ substrate is commonly used for high-yield growth of long SWNTs. Accordingly, we prepared the alumina layer by oxidizing a 15-nm-thick Al layer on a silica substrate in air at $773 \mathrm{~K}$ for $15 \mathrm{~min}$, following the procedure of Ref. 24 .

It is known that SWNTs tend to lie on the substrate surface when the density of metal catalysts is low, while SWNTs tend to grow vertically when the catalyst density is high. ${ }^{16)}$ In order to synthesize A-CNT and R-CNT films, Co catalysts were supported on the alumina layer by vacuum evaporation and dip-coating, respectively, because the density of the catalysts supported by the former method is much higher than that by the latter method. In the former case, Co films of thickness $0.5 \mathrm{~nm}$ were deposited in a vacuum chamber and oxidized fine particles were formed by heating in air at $773 \mathrm{~K}$ for $15 \mathrm{~min}$. In the latter case, Co-containing compounds were coated by the dip-coating procedure ${ }^{17)}$ and oxidized fine particles were formed by heating in air at $673 \mathrm{~K}$ for $5 \mathrm{~min}$. In the CVD process, $\mathrm{Ar} / \mathrm{H}_{2}$ gas $\left(3 \% \mathrm{H}_{2}\right.$ by volume) flowed inside a quartz tube during heating to the reaction temperature in order to activate the oxidized Co particles. After the furnace temperature reached $973-1073 \mathrm{~K}, \mathrm{Ar} / \mathrm{H}_{2}$ gas was replaced with ethanol vapor and CVD was performed at 4 $\mathrm{kPa}$ for $10 \mathrm{~min}$. CNT samples were then characterized with a scanning electron microscope (SEM; JSM-7001F, JEOL), or a Raman spectrometer (inVia Reflex, Renishaw) with $532 \mathrm{~nm}$ laser excitation.

Figure 1 shows typical SEM images of an A-CNT film. Figures 1(a)-1(e) are taken with the alumina substrate tilted by $70^{\circ}$ from the position for the top view so that the crosssectional view is highlighted. The flat area in Figs. 1(a) and 1 (c) is the substrate surface of the alumina layer which is exposed due to intentional peeling off of parts of the CNT film. Since the CNTs grow sparsely because of the lack of catalyst particles with smaller diameters favorable for CNT growth, ${ }^{25)}$ the substrate surface is partially exposed in Fig. 1(e). As shown there, films with a thickness of about 10 and $0.5 \mu \mathrm{m}$ for A-CNT and R-CNT, respectively, were employed in the SHG measurements.

\subsection{SHG experiments}

Figure 2 shows an experimental setup for SHG measurements and has been described elsewhere. ${ }^{26)}$ The linearly polarized laser radiation at $1064 \mathrm{~nm}(1.17 \mathrm{eV})$ from a $Q$-switched Nd: YAG laser with a repetition rate of $10 \mathrm{~Hz}$, a pulse duration of $10 \mathrm{~ns}$ and a bandwidth of $0.3 \mathrm{~cm}^{-1}$ was directed at an angle of incidence of about $45^{\circ}$ onto the sample surface and weakly focused to a diameter of $1.5 \mathrm{~mm}$. The $\mathrm{SHG}$ signal was detected by a photomultiplier tube with gated electronics averaging for typically 100 pulses. The input laser energy was less than $1.5 \mathrm{~mJ}$ per pulse; this was low enough to prevent any irreversible changes from being induced on the surface. In the experiment, we measured the polarizationselected SHG intensity $G_{\mathrm{pp}}$ and $G_{\mathrm{sp}}$, where $G_{i j}$ indicates the $j$ polarized SH intensity for an $i$-polarized pump field and the polarization-selected SHG ratio $\left(G_{\mathrm{pp}} / G_{\mathrm{sp}}\right)$ was used in the data analysis. Note the incident beam with $s$ or $p$ polarization is allowed to generate $\mathrm{SH}$ photons with $p$ polarization even on an isotropic surface through a different component of the second-order susceptibility. ${ }^{18)}$

UV laser irradiation on CNT films is known to increase the threshold for laser-induced damage, probably because the irradiation removes carbon impurities from the films. ${ }^{27)}$ Accordingly, all the SWNT samples were irradiated with the fourth harmonic wave of the Nd:YAG laser $(266 \mathrm{~nm})$ at a power of $80 \mathrm{~mW}$ for $30 \mathrm{~s}$ in an $\mathrm{Ar} / \mathrm{H}_{2}$ atmosphere, increasing the threshold for laser-induced damage by two orders of magnitude.

\section{Results and discussion}

A typical Raman spectrum of as-grown A-CNT film is shown by the dotted curve in Fig. 3(a). Prominent peaks at $1575 \mathrm{~cm}^{-1}$ and $1335 \mathrm{~cm}^{-1}$ are usually assigned to the G-band characteristic of the nanotube carbon and the D-band characteristic of non-nanotube carbon impurities, respectively. ${ }^{4)}$ As shown in Fig. 3(b), we also observed some sharp lines between 150 and $350 \mathrm{~cm}^{-1}$. They are assigned as the radial breathing mode (RBM), which is unique to SWNTs with a tube diameter $d$ in the range $0.7 \mathrm{~nm}<d<2 \mathrm{~nm}^{4}{ }^{4}$

The Raman spectrum of an A-CNT film cleaned by UV laser irradiation ${ }^{27)}$ is shown by the solid line in Fig. 3(a). The SEM images are also shown in Fig. 3(c). It seems that bundles of CNTs are covered by some dark layer before irradiation, while they are more clearly seen after irradiation. Correspondingly, after cleaning by UV laser irradiation, the intensity of the D-band peak was decreased by $25 \%$ and the intensity ratio G/D of the G-band peak intensity to the Dband peak intensity became 3.38 , indicating a high crystalline quality. ${ }^{4)}$ In addition, a shoulder of the G-band at $1550 \mathrm{~cm}^{-1}$ is known to represent evidence for the presence of electrically metallic CNTs. ${ }^{4)}$ Thus A-CNT films may naturally be a mixture of metallic and semiconducting CNTs.

The diameter of CNTs can be estimated from RBM peaks. Especially in the CNTs synthesized by CVD method, the angular frequency of RBM, $\omega_{\mathrm{RBM}}$ in units of $\mathrm{cm}^{-1}$, is 

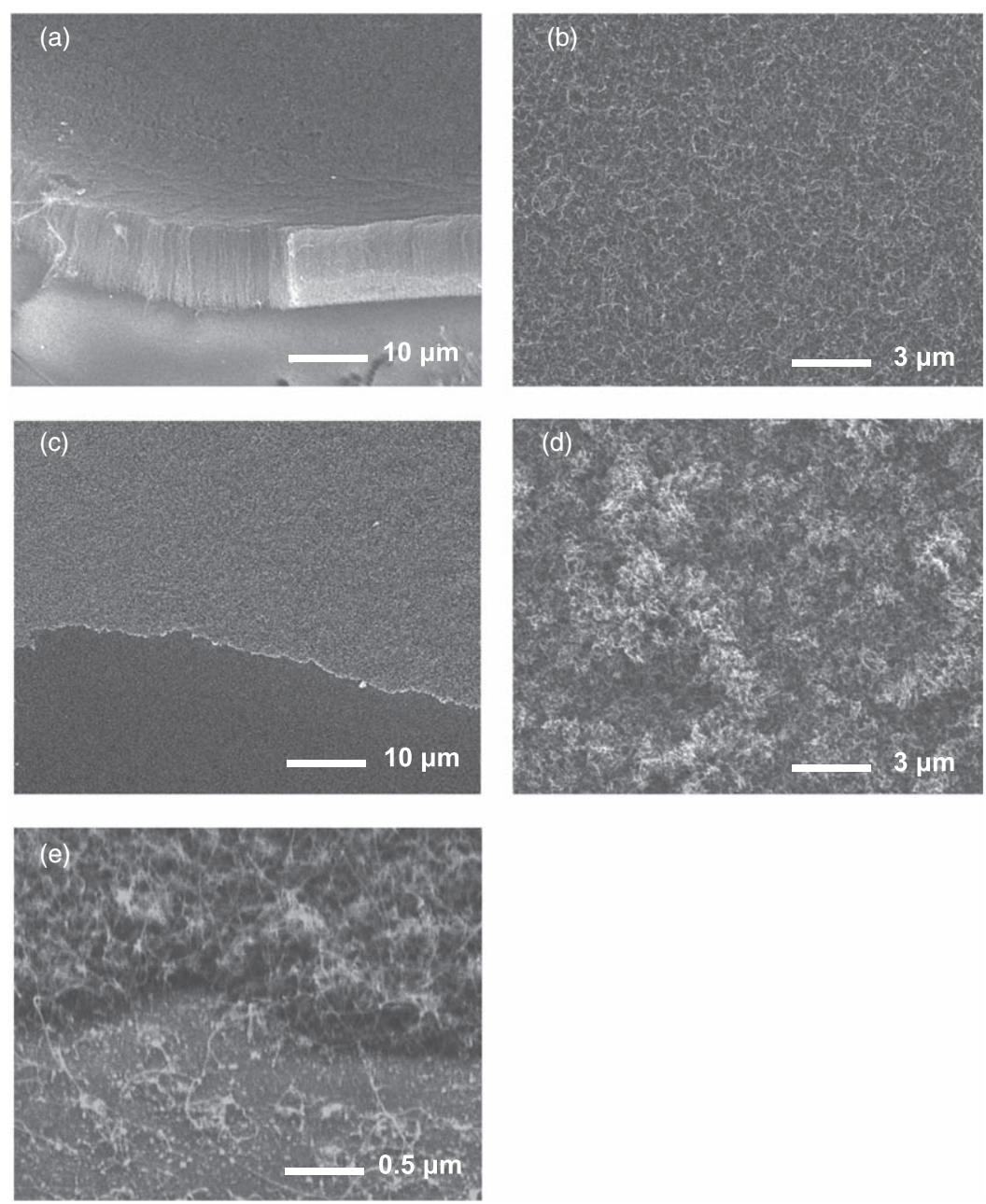

Fig. 1. SEM images of A-CNT and R-CNT: (a) cross-sectional view of A-CNT on alumina, (b) top view of A-CNT, (c) cross-sectional view of R-CNT on alumina, (d) top view of R-CNT, (e) magnified cross-sectional view of R-CNT. Parts (a), (c) and (e) were taken with the alumina substrate tilted by $70^{\circ}$ from the position for the top view observation. The film thickness was about 10 and $0.5 \mu \mathrm{m}$ for A-CNT and R-CNT, respectively. Flat areas in (a) and (c) are the substrate alumina obtained by accidental removal of the CNTs. The flat area in (e) was formed depending on the density of the catalyst particles (see text).

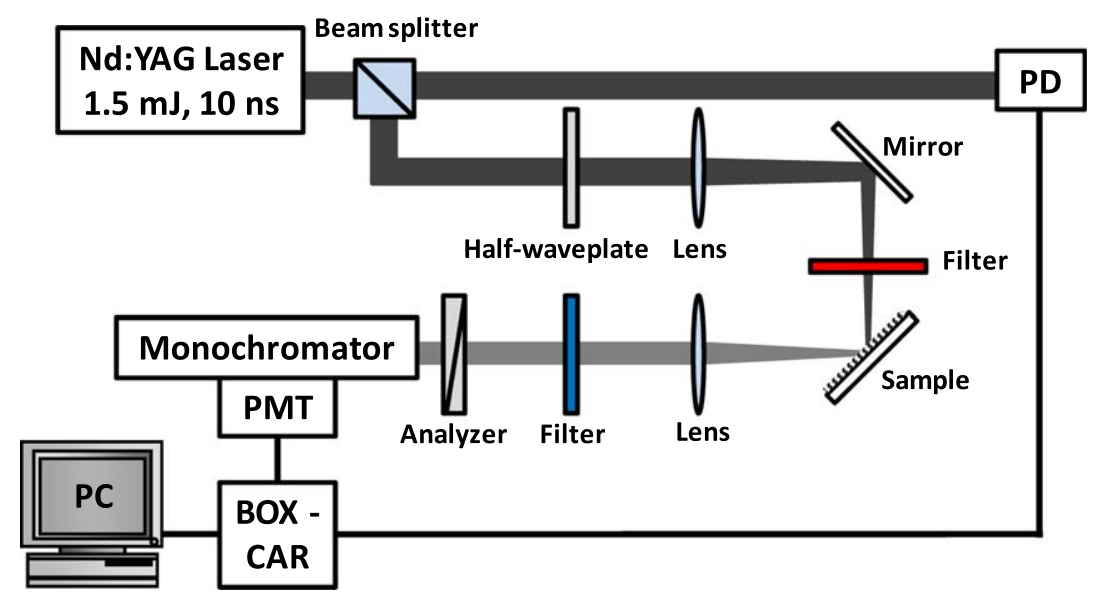

Fig. 2. (Color online) Experimental setup for SHG measurement.

described to be proportional to the reciprocal of the CNT diameter $d$ by the following equation: ${ }^{28)}$

$$
\omega_{\mathrm{RBM}}=248 / d .
$$

Thus, the strongest RBM peak at $220 \mathrm{~cm}^{-1}$ in Fig. 3(b) is attributed to CNTs with $d=1.13 \mathrm{~nm}$. These CNTs are identified to be metallic, because metallic CNTs with $d \sim 1.1 \mathrm{~nm}$ are expected from the Kataura $\operatorname{plot}^{29)}$ to dominate the resonance Raman spectra excited at $532 \mathrm{~nm}$.

Meanwhile, a few relatively broad peaks were observed between 1.10 and $1.75 \mathrm{eV}$ in the photo-absorption spectra (not shown). According to the Kataura plot, these peaks are assigned to those CNTs having $d=0.78 \sim 1.40 \mathrm{~nm}$, which are typical values for CVD-grown CNT film. ${ }^{5,29)}$ Among 
(a)

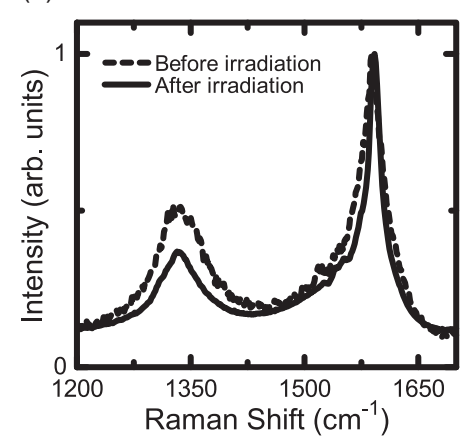

(b)

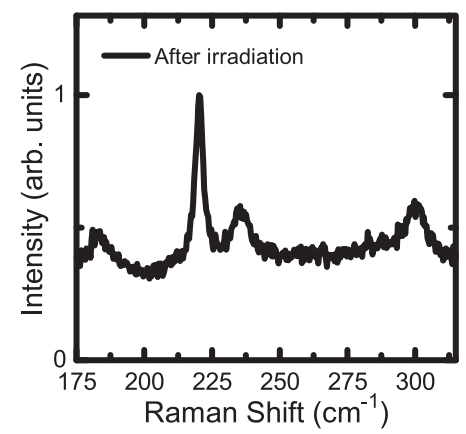

(c)

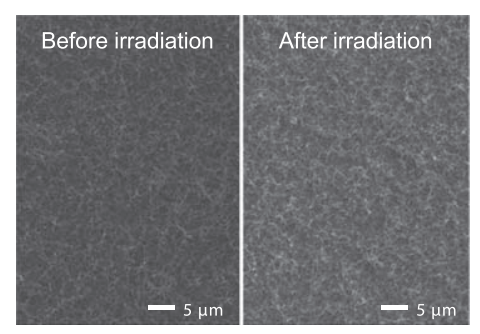

Fig. 3 (a) Raman signal for A-CNT as-grown on alumina (dotted line) and the Raman signal obtained after UV laser cleaning (solid line). Two typical peaks are the G-band and D-band peaks at $1575 \mathrm{~cm}^{-1}$ and $1335 \mathrm{~cm}^{-1}$, respectively. (b) The radial breathing mode peaks at $150-300 \mathrm{~cm}^{-1}$ for A-CNT on alumina. (c) SEM images for A-CNT before and after the UV laser irradiation.

them, semiconducting CNTs having the smallest diameter $(0.78 \mathrm{~nm})$ are expected to make the biggest contribution to SHG, because optical nonlinearity is larger for smallerdiameter CNTs. ${ }^{30)}$

The chiral vector $(n, m)$ of SWCNT was estimated by the formula

$$
d=\frac{a}{\pi} \sqrt{n^{2}+m^{2}+n m}
$$

where $a$ is the lattice constant of graphite $(a=0.246 \mathrm{~nm})$. For the later analysis of $G_{\mathrm{pp}} / G_{\mathrm{sp}}$ according to Ref. 23 , we use as typical chiral vectors $(15,0)$ for metallic CNTs with $d=1.13 \mathrm{~nm}$ and $(10,0)$ for semiconducting CNTs with $d=0.78 \mathrm{~nm}$, because the dielectric constants needed for the analysis for these chiral indices are available in Ref. 12 . Note that both indices show achiral CNTs. Dielectric constants obtained from Ref. 12 are $-4+7 \mathrm{i}$ for $(10,0)$ and $-25+\mathrm{i}$ for $(15,0)$ at a wavelength of $1064 \mathrm{~nm}$, and $6+18 \mathrm{i}$ for $(10,0)$ and $-8+8 \mathrm{i}$ for $(15,0)$ at a wavelength of $532 \mathrm{~nm}$, respectively. These dielectric constants give absorption coefficients larger than $3 \times 10^{7} \mathrm{~m}^{-1}$ at $532 \mathrm{~nm}$ for both indices in the case where the CNT film is entirely either (10, $0)$ or $(15,0)$. This shows that our samples, even though they are a mixture, may be optically thick. These dielectric constants will be used later in our order-of-magnitude estimation of the SHG intensity data.

Figure 4 show the typical polarization-selected SHG intensities as a function of laser fluence for R-CNTs. A quadratic dependence on the laser fluence ascertains the SHG signal. The SHG signal was almost $p$ polarized, the intensity of $s$ polarization being less than the detection limit of our experimental apparatus. The SHG intensity observed for CNT films in the repeated measurements was quite similar and homogeneous, which indicates that the CVD growth conditions were stable enough. The $G_{\mathrm{pp}} / G_{\mathrm{sp}}$ value obtained in Fig. 4 is 2.3 for A-CNT and 1.1 for R-CNT. The ratios depend only slightly on the CVD growth conditions, and these ratios in Fig. 4 may be taken as typical values for the two kinds of film. It may be noted that this ratio is also different from that reported for a smooth metal surface, obtained $^{31)}$ and calculated ${ }^{32)}\left[\mathrm{G}_{\mathrm{pp}} / G_{\mathrm{sp}} \sim 23(=1 / 0.046)\right]$ for afresh silver surface. We also reproduced such a high $G_{\mathrm{pp}} / G_{\mathrm{sp}}$ in another experiment by using a commercial gold mirror.

Four possible sources may be discussed within the electricdipole approximation for the SHG signal in reflection from

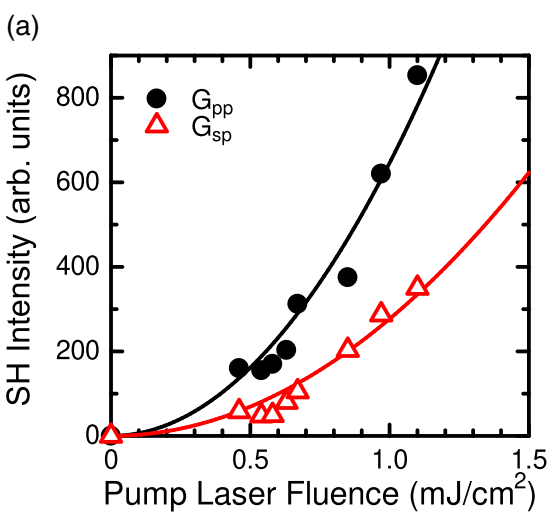

(b)

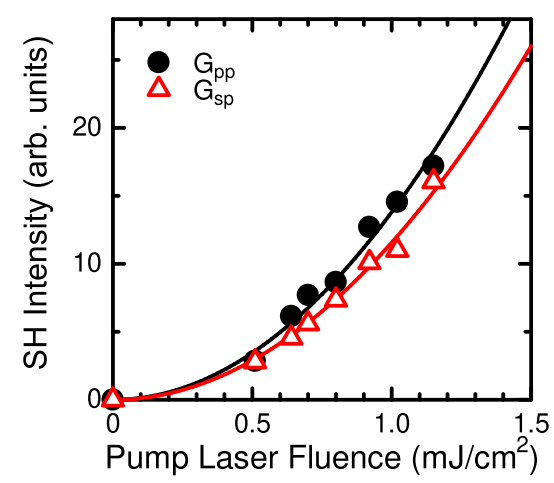

Fig. 4 (Color online) (a) Polarization-selected SHG intensity as a function of laser fluence at an incidence angle of $45^{\circ}$ for $G_{\mathrm{pp}}$ (solid circles) and $G_{\mathrm{sp}}$ (red triangles) for A-CNT. (b) SHG as a function of laser fluence for $G_{\mathrm{pp}}$ (solid circles) and $G_{\mathrm{sp}}$ (red triangles) for R-CNT. The full curve is a quadratic fitting, and $G_{\mathrm{pp}} / G_{\mathrm{sp}}$ is obtained to be about 2.3 for A-CNT and 1.1 for RCNT.

CNT films: chirality, lattice defects, tube deformations of CNTs and surface interactions. ${ }^{5-11,17-21)}$ We discard chirality as the source of SHG, since the SHG signal was almost $p$ polarized for $p$ excitation, leaving the intensity of the $s$ component of SHG contributed by chirality to be negligibly small. ${ }^{18)}$ The negligible intensity for $P_{\text {in }} S_{\text {out }}$ SHG from the aligned CNT film was also interpreted by the absence of chirality due to a racemic mixture. ${ }^{11)}$ Bulk defects of the CNTs are also discarded as the SHG source for the same reason, since the $s$ component of SHG is negligibly small. The quite similar and homogeneous signal intensity repeatedly obtained for various CNT films grown under similar conditions also supports the discarding of lattice defects or 
CNT tube deformations as the source of SHG. Because the CNT films with a thickness greater than $1 \mu \mathrm{m}$ are optically thick enough, we can neglect the contribution of the underlying substrate interface to our SHG signal. Thus, we attribute the observed SHG to the surface: the CNT filmair interface. Then, the surface conditions and the surface morphology may be reflected in the signal, as will be discussed further in the following.

The SEM images in Figs. 1(a) and 1(b) show that A-CNT film may naturally be taken as a collection of differently sized (and differently shaped) nanowires aligned vertically to the surface with the space between the CNTs determined by the catalyst scattering. Under this assumption, each CNT can be described as forming a needle-like protrusion in the case of the A-CNT sample. Thus, we will consider SHG from ACNT films with nanometer-sized protrusions which are either metallic or semiconducting. It may be noted that a needle-like protrusion has a diameter of about $1 \mathrm{~nm}$ and a length corresponding to the penetration depth of the fundamental laser light or the $\mathrm{SH}$ light.

For R-CNT, Figs. 1(c) and 1(d) show a corrugated surface, where differently shaped CNTs appear to bundle together to form composites of differently shaped nanometer-sized protrusions distributed randomly on the substrate surface. From the images the lateral size of the protrusions may be roughly $1 \mu \mathrm{m}$ with a typical separation of $1-2 \mu \mathrm{m}$, which is of the same order as the laser wavelength employed. Thus, the size of the detailed structures inside the protrusions is clearly much smaller than the laser wavelength. Note that the protrusions are a mixture of both metallic and semiconducting CNTs, where protrusions of nanowires with random orientations may not contribute to SHG since the film is macroscopically isotropic and achiral.

There is a long history of attempts to calculate SHG from a film surface with nanometer-sized structures. An ideal case is usually assumed in the calculation, since an exact calculation needing all the structural parameters of the film is unrealistic. A number of works have been devoted to SHG involving clean and flat metal surfaces, ${ }^{32)} \mathrm{Ag}$ particles on a lithographically produced microstructure, ${ }^{33)}$ spherical clusters, ${ }^{34)}$ colloidal films composed of spheroidal particles ${ }^{23)}$ and metal island films. ${ }^{20,35)}$

In the SHG study with island films the polarization intensity ratios, together with enhancement relative to a smooth silver surface, ${ }^{32}$ ) were used to obtain information regarding the surface conditions or the structural parameters of islands. As has been described, ${ }^{22)}$ this may be understood by a simple model assuming a collection of rotational ellipsoids resting on the substrate with their long axis parallel to it. Nonuniform distribution of the electron density across the particle surface, for example by adsorbates or by attachment of the particle to the substrate, may be expected as follows. When a light is shone on this particle/substrate system, different degrees of nonlinear polarization compensation can be assumed, depending on the polarization direction of the incident light. For irradiation with a polarization parallel to the substrate plane ( $s$ polarization), the nonlinear dipolar surface polarizations at opposite particle-surface elements are practically completely compensated due to geometric mirror symmetry. In contrast, irradiated $p$ polarized light has a component normal to the substrate plane. The nonlinear polarizations driven by this component can be different at the top and bottom of the particle as there are different interfaces: metal/outside and metal/substrate, respectively. The works discussed were executed in a system in the colloid ${ }^{23)}$ and in the island film. ${ }^{32)}$

SHG behavior of spheroidal metal particles with respect to the polarization ratio has been developed and applied to model interfacial films of spheroidal particles with varying diameters, $\left.{ }^{23}\right)$ where metal particles dispersed in a nonconductive dielectric medium form not a pure metal (in this case silver) film but a metal liquid-like film. This model considers particles that are much smaller $(<100 \mathrm{~nm})$ than the wavelength of light and are arranged as an array, for example at the interface between two media. On the metal surface, the second-order nonlinear electric susceptibility is dominated by the $\chi_{/ / \perp / /}$ element. ${ }^{19)}$ Here, subscripts // and $\perp$ represent two tangential components and the normal component on the spheroid surface, respectively. The $\chi_{/ / \perp / /}$ of an individual particle's surface is calculated and averaged susceptibilities $\chi_{\mathrm{ijk}}$ are derived by projecting $\chi_{/ / \perp / /}$ onto $\chi_{\mathrm{ijk}}$ and integrating over the hemi-ellipsoid surface. The subscripts $i, j, k$ are either $x$ or $y$ or $z$. The eccentricity is accordingly described by $x^{2}+y^{2}+(z / \eta)^{2}=1$, where $x, y$ and $z$ are normalized to the radius of the circular projection of the ellipsoid on the $x y$ plane and $\eta$ is the eccentricity. The usual model of a 'polarization sheet' is employed in the SHG analysis. ${ }^{36)}$ Dispersed colloidal films at low concentrations are assumed and the SHG results are analyzed without incorporating the electromagnetic interaction between the particles. Using the spheroidal model and the eccentricities, the polarization ratio of SHG was derived for such a 'composite' interface, which is macroscopically uniform but composed of nanometer-sized silver particles suspended in a very different dielectric medium. The shape of spheroidal particles is thus connected to $G_{\mathrm{pp}} / G_{\mathrm{sp}}$, which is the reflected $\mathrm{SHG}$ intensity ratio. According to the explicit calculation in Ref. 23, $G_{\mathrm{pp}} / G_{\mathrm{sp}}$ is given by the following equation:

$$
\begin{aligned}
G_{\mathrm{pp}} / G_{\mathrm{sp}}= & \mid 1 / 2 k_{1}[2-C(\eta)]+1 / 2 k_{2} C(\eta) \\
& -1 /\left.4 k_{3} C(\eta)^{2}\right|^{2} /\left|1 / 4 k_{4} C(\eta)\right|^{2},
\end{aligned}
$$

with

$$
C(\eta)=2 \eta^{2}\left[\eta^{2}-1-2 \ln \eta\right] /\left(\eta^{2}-1\right)^{2} .
$$

Coefficients $k_{i}$, the functions of the incidence angle of laser light and the complex dielectric constants at both the fundamental and harmonic frequencies, are calculated according to published formulae. ${ }^{22,36)}$ The above treatment may be extended to the dielectric particles by simply replacing the dielectric functions.

We notice that our corrugated sample may also be approximated by this model, where the condition of dispersed colloidal films (at low concentrations) may be automatically fulfilled by the fact that SWNT or CNT protrusions (or islands) are not pure metal but a mixture of metal and dielectric. In order to interpret the dependence of SHG polarization ratios obtained for the CNT samples, we model each CNT sample by the spheroidal model with the eccentricities, so that numerical estimation is possible within the framework described above.

Figure 5 shows $G_{\mathrm{pp}} / G_{\mathrm{sp}}$ as a function of eccentricity, calculated for a model system of CNTs with chiral indices of 


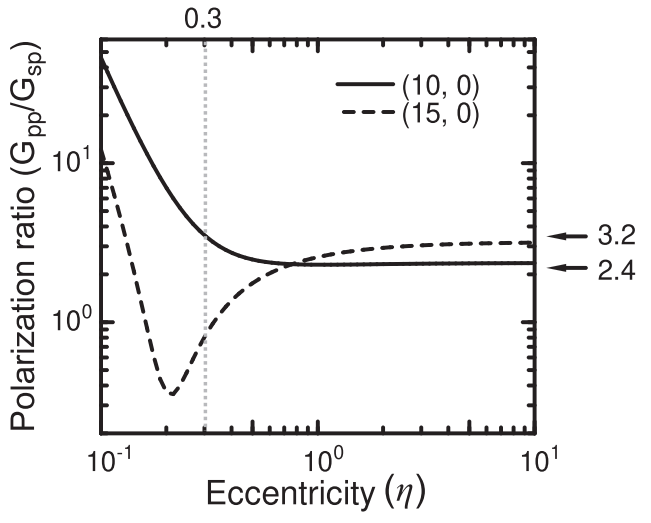

Fig. 5 The polarization intensity ratio $\left(G_{\mathrm{pp}} / G_{\mathrm{sp}}\right)$ as a function of eccentricity $\eta$, calculated for a model system for CNTs with chiral indices of (15, $0)$, which metallic, and $(10,0)$, which is semiconducting. Note that both indices show achiral CNTs.

$(15,0)$, which is metal, and $(10,0)$, which is semiconducting. The singularity at an eccentricity of 0.2 in the case of a metal is also reproduced, although not mentioned clearly in Ref. 23; this singularity missing in the semiconducting CNT may be related to localized or particle plasmon excitation. ${ }^{33)}$ Note that this singularity causes a very large scattering in $G_{\mathrm{pp}} / G_{\mathrm{sp}}$ in the adjacent eccentricity range, depending rather strongly on both the eccentricity and the metallicity.

The A-CNT described here is a sparsely distributed needlelike protrusion. It may be noted that the needle-like protrusions have a diameter of about $1 \mathrm{~nm}$ and a length equal to the penetration depth of the fundamental laser light or its $\mathrm{SH}$. The absorption coefficient of $8 \times 10^{7} \mathrm{~m}^{-1}$ at $532 \mathrm{~nm}$ will give a typical penetration depth of about $13 \mathrm{~nm}$. Thus, the top part (about $13 \mathrm{~nm}$ long) of CNT needle will contribute to SHG. A CNT about $13 \mathrm{~nm}$ long and with a radius of about $1 \mathrm{~nm}$ will give roughly $\eta \sim 13 . G_{\mathrm{pp}} / G_{\mathrm{sp}}$ is calculated to be 3.2 for $(15,0)$ and 2.4 for $(10,0)$, respectively. The experimental data for $G_{\mathrm{pp}} / G_{\mathrm{sp}}=2.3$ is thus roughly consistent with the estimation. Note that metallic or semiconducting character may not make a large difference in the region where the eccentricity is large.

For R-CNT, shown in Fig. 1(d), CNTs bundle together to form composites of differently shaped nanometer-sized protrusions randomly distributed on the substrate surface with a typical lateral size of roughly $1 \mu \mathrm{m}$ and a separation of 1-2 $\mu \mathrm{m}$. The film thickness of R-CNT shown in Fig. 1(e), a projected image in the normal direction of the substrate with a projection correction parameter of $1 / \sin 70^{\circ}(\sim 1.06)$, may be estimated to be $0.5 \mu \mathrm{m}$ with a corrugation of about half the thickness. Hence, the height of corrugation is $\sim 0.3 \mu \mathrm{m}$, i.e. about half the film thickness of $0.5 \mu \mathrm{m}$. If we assume in our order-of-magnitude estimation the same dielectric constant for R-CNT as for A-CNT, a penetration depth of $\sim 13 \mathrm{~nm}$ may also be expected for R-CNT; this is negligibly small compared with the height of corrugation. Thus, SHG does not probe the details inside the protrusions but instead probes the contour of the protrusions from the R-CNT film, i.e. the surface of nanometer-sized protrusions composed of a mixture of metallic and semiconducting CNTs. A typical eccentricity of $\eta \sim 0.3$, corresponding to a protrusion height of $0.3 \mu \mathrm{m}$, may be assumed for R-CNT, which gives a $G_{\mathrm{pp}} / G_{\mathrm{sp}}$ in Fig. 5 of 0.8 for $(15,0)$ and 3.5 for $(10,0)$, respectively. Note that the dielectric constants of the actual film cannot clearly be described by those of the pure materials, reflecting the fact that the naturally formed film is a mixture of metal and semiconducting CNTs. Since the experimental data for $G_{\mathrm{pp}} / G_{\mathrm{sp}}=1.1$ exists within the above scattering range, one could expect that the model calculation may reproduce the data, given detailed information on the surface morphology and the composition of metal and semiconducting nanotubes. It is thus shown that the polarization ratio of SHG may discriminate between A-CNT and R-CNT through the eccentricity representing the surface morphology.

Since the topmost region monitored by SHG is as shallow as $\sim 10 \mathrm{~nm}$ in the A-CNT and R-CNT films, the difference in the surface morphology in the initial stage of CNT growth may also be reflected in the polarization ratio. Note that the installation of proper windows for laser light guides in the CVD system for remote sensing may be accomplished with ease. The present finding may thus be applied to in situ evaluation of the CVD growth process by connecting $G_{\mathrm{pp}} / G_{\mathrm{sp}}$ to the surface morphology and surface conditions during the initial stage of formation of CNTs under CVD. For this purpose, it may also be crucial to increase the achievable signal-to-noise ratio by employing a higher excitation power and shorter pulses delivered by a mode-locked laser; this would also be desirable from the point of view of sample damage and better stability with a wider wavelength tuning range. Another possibility would be to perform measurements closer to resonant conditions, either by tuning the excitation wavelength or by selecting different types of SWNTs.

\section{Conclusions}

In summary, we have studied the polarization dependence of surface SHG from CNT films supported on a $\mathrm{SiO}_{2}$ substrate prepared by in-house CVD. The SHG intensity for the incident radiation polarized perpendicular or parallel to the plane of incidence at the fundamental radiation $(1064 \mathrm{~nm})$ and its intensity ratio is observed to have typical values for the two kinds of grown films - one an aligned CNT film and the other a film of randomly oriented CNTs lying on a substrate sheet. By approximating the CNT-air interface of CNT films by a composite film of spheroidal particles with varying diameters, we were able to calculate and qualitatively reproduce the observed polarization ratios. SHG is found to originate not from the CNT bulk but from the film surface; moreover, we show that the surface morphology of the CNT film is reflected in the polarization ratio. The present finding is also proposed to be applicable to in situ evaluation of the initial stage in the CVD growth process of CNTs.

\section{ORCID iDs}

Masatoshi Tanaka (iD https://orcid.org/0000-0001-8252-0971 Takanori Suzuki (iD https://orcid.org/0000-0003-3418-6395

1) S. Iijima, Nature 354, 56 (1991).

2) P. Avouris, Z. Chen, and V. Perebeinos, Nat. Nanotechnol. 2, 605 (2007).

3) M. F. L. De Volder, S. H. Tawfick, R. H. Baughman, and A. J. Hart, Science 339, 535 (2013).

4) M. S. Dresselhaus, G. Dresselhaus, R. Saito, and A. Jorio, Phys. Rep. 409, 47 (2005).

5) Y. Miyauchi, S. Chiashi, Y. Murakami, Y. Hayashida, and S. Maruyama, Chem. Phys. Lett. 387, 198 (2004). 
6) H. M. Su, J. T. Ye, and K. S. Wong, Phys. Rev. B 77, 125428 (2008).

7) S. O. Konorov et al., J. Raman Spectrosc. 34, 1018 (2003).

8) D. A. Akimov et al., Laser Phys. 13, 1279 (2003).

9) S. Botti, R. Ciardi, L. De Dominicis, L. Asilyan, R. Fantoni, and T. Marolo, Chem. Phys. Lett. 378, 117 (2003).

10) L. De Dominicis, S. Botti, L. S. Asilyan, R. Ciardi, R. Fantoni, M. L. Terranova, A. Fiori, S. Orlanducci, and R. Appolloni, Appl. Phys. Lett. 85, 1418 (2004).

11) L. De Dominicis, R. Fantoni, S. Botti, R. Ciardi, L. Asilyan, A. Fiori, and S. Orlanducci, J. Raman Spectrosc. 36, 165 (2005).

12) G. Y. Guo, K. C. Chu, D. S. Wang, and C. Duan, Phys. Rev. B 69, 205416 (2004).

13) M. J. Huttunen et al., New J. Phys. 15, 083043 (2013).

14) M. Kumar and Y. Ando, J. Nanosci. Nanotechnol. 10, 3739 (2010).

15) T. Belin and F. Epron, Mater. Sci. Eng. B 119, 105 (2005).

16) S. Maruyama, R. Kojima, Y. Miyauchi, S. Chiashi, and M. Kohno, Chem. Phys. Lett. 360, 229 (2002).

17) Y. Murakami, S. Chiashi, Y. Miyauchi, M. Hu, M. Ogura, T. Okubo, and S. Maruyama, Chem. Phys. Lett. 385, 298 (2004).

18) Y. R. Shen, The Principles of Nonlinear Optics (Wiley, New York, 1984).

19) C. K. Chen, T. F. Heinz, D. Ricard, and Y. R. Shen, Phys. Rev. B 27, 1965 (1983).

20) T. Miyamae, Y. Miyata, and H. Kataura, J. Phys. Chem. C 113, 15314 (2009).

21) F. R. Aussenegg, A. Leitner, and H. Gold, Appl. Phys. A 60, 97 (1995).
22) R. Bavli, D. Yogev, S. Efrima, and G. Berkovic, J. Phys. Chem. 95, 7422 (1991).

23) G. Berkovic and S. Efrima, Langmuir 9, 355 (1993).

24) H. Ohno, D. Takagi, K. Yamada, S. Chiashi, A. Tokura, and Y. Homma, Japan. J. Appl. Phys. 47, 1956 (2008).

25) S. Noda, H. Sugime, T. Osawa, Y. Tsuji, S. Chiashi, Y. Murakami, and S. Maruyama, Carbon 44, 1414 (2006).

26) T. Suzuki, Phys. Rev. B 61, R5117 (2000).

27) K. E. Hurst, A. C. Dillon, D. A. Keenan, and J. H. Lehman, Chem. Phys. Lett. 433, 301 (2007).

28) A. Jorio, R. Saito, J. Hafner, C. M. Lieber, G. Dresselhaus, and M. S. Dresselhaus, Phys. Rev. Lett. 86, 1118 (2001).

29) H. Kataura, Y. Kumazawa, Y. Maniwa, I. Uemezu, S. Suzuki, Y. Ohtsuka, and Y. Achiba, Synth. Met. 103, 2555 (1999).

30) H. E. Ruda and A. Shik, J. Appl. Phys. 101, 034312 (2007).

31) F. Brown and M. Matsuoka, Phys. Rev. 185, 985 (1969).

32) J. Rudnick and E. A. Stern, Phys. Rev. B 14, 4274 (1971).

33) A. Wokaun, J. G. Bergman, J. P. Heritage, A. M. Glass, P. F. Liao, and D. H. Olson, Phys. Rev. B 24, 849 (1981).

34) J. Dadap, J. Shan, and T. F. Heinz, J. Opt. Soc. Am. B 21, 1328 (2004).

35) T. Suzuki, K. Fujiwara, A. Kokabu, T. Momose, H. Okamoto, and M. Tanaka, Appl. Surf. Sci. 267, 58 (2013).

36) V. Mizrahi and J. E. Sipe, J. Opt. Soc. Am. B 5, 660 (1988). 